\title{
Die Notwendigkeit neuer Leitbilder - Plädoyer für eine ökologisch orientierte Wirtschaftspolitik in den neuen Bundesländern
}

\begin{abstract}
Mittlerweile wird vom deutschen Bundeskanzler zugestanden, daß die „, blühenden Landschaften “ sich noch im Wartestand befinden und daß es mit einfacher Subventionierung und dem Vertrauen auf die Wachstumskräfte der Marktwirtschaft allein nicht getan ist. Daß man dies direkt nach der Vereinigung auf Regierungsseite nicht wahrnehmen wollte und darauf bis heute nicht angemessen reagiert hat, dürfte eines der Hauptprobleme der gegenwärtigen gesellschaftlichen Verdrossenheit in Ost und West darstellen. Züdem ist festzustellen, daß der materiell gesättigte Westen, nicht allein in Deutschland, sondern auch in der EG beim Auftreten von rezessiven Tendenzen seine Containance verliert. Nachdem das östliche System abgewirtschaftet hat, verliert der Westen seine offenbar zentrale Orientierung. Sie bestand - wie immer deutlicher wird - im wesentlichen in der Abgrenzung zum östlichen System. Die Haltlosigkeit der rein materiellen Wertorientierung im Westen wird deutlich, wenn mit der Begrenzung der Wachstumsaussichten die gesellschaftlichen Befriedungsaktionen in Bedrängnis geraten. Dies zum einen in sozialer Hinsicht - mit dem im nächsten Jahr anstehendem Haushaltssicherungsgesetz werden ,notwendige' Schnitte in das soziale Netz vorgenommen werden, sowie in ökologischer Hinsich, wenn jetzt der von der Industrie geäußerte Widerstand gegen weitere Verschärfungen der Umweltgesetzgebung immer stärker wird.
\end{abstract}

Im Jahre drei nach der Wirtschafts- und Währungsunion werden nun die Warnungen vor einer massiven Deindustrialisierung in den neuen Bundesländern lauter - zu einem Zeitpunkt, da dieser Prozeß zumindest kurzfristig kaum aufhaltbar zu sein scheint. Kurzfristig nicht aufhaltbar vor allem aus folgenden Gründen:

- Zusammenbruch der Produktion, insbesondere im Bereich der Investitionsgüter; hier lag die Produktion Mitte 1992 um 2/3 niedriger lag als vor der Wende.

- Zumindest mittelfristiger Zusammenbruch des Osthandels. Gegenwärtig stehen in diesem Bereich noch 400.000-600.000 Arbeitsplätze auf dem Spiel, und dies trotz der Gewährung der Hermes-Kredite, die der Situation in den mittel- und osteuropäischen Ländern allerdings z. T. nicht mehr angepaßt sind;

- Erheblichen konjunkturelle und strukturelle Schwierigkeiten in der westdeutschen Industrie, damit bedrohte Neuinvestitionen in den neuen Bundesländern;

- Ausbleibende Erholung der Weltkonjunktur insbesondere in den für die neuen Bundesländer zentralen Bereichen Werkzeugmaschinenbau, Automobil, Chemie und Stahl.
Insbesondere die letztgenannten Faktoren führen dazu, daß die nicht wettbewerbsfähigen Unternehmen in Ostdeutschland weiter unter Druck geraten werden und es auch für die Treuhand und die Wirtschaftspolitik immer schwieriger wird, in dieser Situation gegenzusteuern. Sie treten neben die bereits kurz nach der Währungsunion eingeleiteten Entwicklungen, die bereits erhebliche Auswirkungen auf die Wirtschaft der neuen Länder hatten:

- Die Unternehmen in den neuen Bundesländer wurden, so sie nicht schnell privatisiert werden konnten, in den Wartestand entlassen. Ein Großteil der Treuhandunternehmen hat nun das dritte Jahr hinter sich, ohne daß entscheidende Umstrukturierungen vorgenommen worden sind; dies nicht zuletzt aufgrund der fehlenden finanziellen Ausstattung. Die Folge ist, daß sich die Konkurrenzverhältnisse kontinuierlich verschlechtern.

- Es sind zwar Mittel in erheblichem Umfang in die Unternehmen geflossen, diese sind allerdings nur in geringem Umfang für investive Maßnahmen bereitgestellt worden, so daß der Zustand der Lethargie nicht überwunden werden konnte.
- Die Privatisierung und der Aufkauf durch westliche Unternehmen führte zu radikalen Umstrukturierungen und Freisetzungen von Mitarbeitern sowie zur Einpassung in das Produktionsprogramm des jeweiligen Unternehmens. Dabei war bei westdeutschen Aufkäufern durchaus auch ein entscheidendes Motiv, auf dem deutschen Markt keine Konkurenz für sich entstehen zu lassen.

- In der aktuellen Situation sind die Schwierigkeiten für die noch zu privatisierenden Unternehmen sehr hoch, da eine Umstrukturierung der Produktion und der Produkte in den Unternehmen erfolgen muß, die dann gegen eine starke westliche Konkurrenz antreten müssen. Diese versucht aber selbst bzw. muß versuchen, ihre Märkte zu halten bzw. auch noch auszudehnen, um verlorene Märkte wieder zu erobern.

\section{Aus dem Inhalt}

Industrielle Beziehungen und Ökologie unter veränderten Rahmenbedingungen Eckart Hildebrandt

Methodische Überlegungen zur

Ökobilanzierung

Hans-Knud Arndt

Übersicht über Produkt-Bilanzen Frieder Rubik

Hochschulserie

Weiterbildungsstudium „Umweltmanagement" für betriebliche

Praktiker

Klaus Tischler

Tagungsberichte

Gesellschaft für berufliche Umweltbildung - Rückblick und Ausblick

Christoph Nitschke

INFORMATIONSDIENST 1992

Jahresübersicht

Rezensionen 


\section{Konsequenz für die Beschäftigungssituation}

Die Beschäftigungssituation in den neuen Ländern ist dramatisch: Von den einst 9,8 Mio. Beschäftigten sind mittlerweile lediglich 5,8 Mio. noch in ,normalen 'Beschäftigungsverhältnissen, zusätzlich sind 390.000 in staatlich gestützten Beschäftigungsverhältnissen. Könnten die westdeutschen Verhältnisse übertragen werden, so würden rund 6,5 Mio Menschen beschäftigt werden können. Aber bis zu einer Annäherung an solche Relationen stehen noch erhebliche Anpassungen bevor und es wird sehr viel Zeit kosten, ähnliche Verhältnisse zu erreichen. Verschärfend kommt hinzu:

- die Freisetzungen durch die Unternehmen der Treuhand sind noch nicht abgeschlossen,

- bei den privatisierten Unternehmen ist weiterhin ein erheblicher Beschäftigungsabbau geplant,

- privatisierte Unternehmen können die scheinbar vertraglich abgesicherten Beschäftigungszusagen nicht einhalten, wobei die Sanktionsmöglichkeiten der Treuhand sich als stumpf erweisen,

- die AB-Maßnahmen laufen aus und die Auseinandersetzungen um die Mittelvergabe werden härter,

- aus dem Bereich der MBOs, die als ein Ausweg aus der Krise angesehen wurden, kommen erste deutliche Warnungen hinsichtlich einer breiten Gefährdung dieses Bereiches.

Um eine Einschätzung des für die Angleichung des Sozialprodukts pro Kopf notwendigen Anpassungszeitraums zu geben, sei hier eine Berechnung von Horst Siebert wiedergegeben: Danach beträgt das Sozialprodukt je Kopf 1991 in Ostdeutschland 12.100 DM im Vergleich zu 41.100 DM im Westdeutschland, also $29 \%$ des westdeutschen Niveaus. Bei einer realen Wachstumsrate von $2 \%$ im Westen und $7 \%$ im Osten werden zur Angleichung dieser Verhältnisse 21 Jahre gebraucht. Bei höheren Wachstumsraten würden sich die Anpassungszeiträume verkürzen, allerdings ist zu berücksichtigen, daß über einen so langen Zeitraum derartige Wachstumsarten noch nicht beobachtet wurden. $\mathrm{Da} B$ hinter solchen Wachstumsraten zudem keine ökologisch orientierte Strategie steht, versteht sich von selbst.

Zusammengefaßt wird nach Einschätzung von Politikern die wirtschaftliche Erholung mittelfristig möglich sein; die Angleichung der Wirtschaftsleistung an das westliche Niveau wird im Jahre 2010 erwartet. Ein „Problem“ sind hingegen die 3-4 Mio. Menschen, die dauerhaft nicht in den Arbeitsmarkt zurückfinden werden. Dementsprechend droht eine soziale Katastrophe in den neuen Ländern, die häufig in der Perspektivendiskusssion ausgeblendet wird. Für die neuen Länder bedeutet dies, daß es in mehrfacher Hinsicht zu Segmentierungen kommen wird:
Es wird eine große Anzahl von Dauerarbeitslosen geben, zu denen ganz überwiegend Frauen und Ältere gehören werden.

- Es wird Regionen geben, die den Anschluß auch auf Dauer nicht finden werden - sozusagen ,Zonen'randgebiete völlig anderen Ausmaßes, in denen bereits heute deutliche Entvölkerungstendenzen festzustellen sind.

\section{Die Umwelt und die Vereinigung}

Der Fall der Mauer hatte direkte Konsequenzen für die umweltpolitische Diskussion in der Bundesrepublik. Programme wie z. B. Fortschritt 90 der SPD hatten den ökologischen Umbau der Gesellschaft auf ihre Fahnen geschrieben, und zunächst wurden diese umweltpolitischen Zielvorstellungen auch noch in den Vereinigungsproze $\beta$ mit hinübergerettet. $\mathrm{Zu}$ diesem Zeitpunkt bestand allerdings noch die Vorstellung, daß die Portokasse zur „Übernahme" der DDR ausreiche. Diese aus dem bis dahin erreichten Konsens geprägten Vorstellungen bleiben spätestens jetzt auf der Strecke, wo festzustellen ist,

- daß der Anschluß teuerer wird und zu Einkommensverlusten auch in Westdeutschland führt,

die international sich verschlechternden Rahmendaten zu der Diskussion um den Standort Deutschland führen,

dies wiederum zur Ablehnung von umweltorientierten Sonderwegen von Seiten der Unternehmensvertreter führt und

gegenläufige Tendenzen wie die Forderung nach Deregulierung und Beschleunigung in allen Bereichen (Genehmigungsverfahren etc.) hervorruft.

Die vom Umweltministerium und auch von Seiten der $\mathrm{EG}$ avisierte $\mathrm{CO}_{2}$ - bzw. Energieabgabe sowie die in der Bundesrepublik vorgeschlagene Abfallabgabe scheinen den allgemeinen wirtschaftspolitischen Problemen zum Opfer zu fallen und dürften bestenfalls zur Finanzierung der Etats wieder hervorgeholt werden. Dann allerdings nur unter dem Finanzierungsaspekt und nicht für den Einsatz im Rahmen einer ökologischen Wende. Und so hat Umweltminister Töpfer offenbar auch ganz erhebliche Schwierigkeiten, seine Vorstellungen durchzusetzen - u. a. aufgrund fehlender Finanzmittel und der Infragestellung des Standortes. In Diskussionsbeiträgen macht Töpfer denn auch klar, woher der Wind im Bundeskabinett weht; nämlich daß es mit der Zunahme der Vereingungsprobleme und der konjunkturellen Entwicklungen zu einer Infragestellung umweltorientierter Maßnahmen kommt. Die Industrievertreter lehnen jede Art von Kostenbelastungen mit dem Hinweis auf die Standortfrage ab; dies gilt insbesondere für kostenträchtige Maßnahmen des Umweltschutzes.

Weiterhin nimmt der allgemeine politische Druck ab, da zum einen die Umweltbewegung als solche - nicht zuletzt aufgrund der politischen Wandlung und der damit einhergehenden veränderten Gewichtung des Umweltthemas an Schlagkraft eingebüßt; zum anderen fällt der Widerstand nicht zuletzt aufgrund der Vielzahl der inganggesetzten Infrastrukturprojekte in den neuen Bundesländer schwer.

\section{Wirtschaftsförderung in den neuen Bundesländern}

Die Art der bislang betriebenen Wirtschaftsförderung hat erhebliche verteilungspolitische Implikationen, da die Förderung via Investitionszuschuß und insbesondere die Investitionszulagen - nach dem Windhundverfahren - vor allem kapitalintensive westliche Großunternehmen bevorteilt. Dies zum einen aufgrund der Förderung von Großinvestitionen, die erheblichen Kapitaleinsatz erfordern, der in der Regel von Bürgern der ehemaligen DDR nicht geleistet werden kann, zum anderen durch die Fokussierung auf die Prestigeobjekte der Großunternehmen, in die ein erheblicher Teil der Investitionszulagen fließt, die per Antrag zugewiesen werden. $\mathrm{Da}$ damit nur geringe $\mathrm{Be}$ schäftigungseffekte erzielt werden, ist bei der Kapitalintensität der teilweise modernsten Produktionen nicht überraschend: Der Kapitaleinsatz je Arbeitsplatz liegt z. T. deutlich über dem in der westdeutschen Industrie. Verwiesen wird dabei zwar auf die indirekten Effekte, die sich aus der Ansiedlung von Zulieferern ergeben (können). Die Effekte sind allerdings in der Regel schwer überprüfbar. Folgende Anmerkungen sind dazu zu machen:

- Die Unternehmen entwickeln sich als Kathedralen in der Wüste, d.h. sie sind mit nur geringen wirtschaftlichen Verflechtungen mit dem Umland und insbesondere mit nur geringen FuE-Kapazitäten und Leitungskompetenzen ausgestattet.

- Die Mittel für die Investitionen fließen als Nachfrage vorrangig nach Westdeutschland und haben Beschäftigungswirkungen allenfalls im Bausektor in den neuen Bundesländern. Damit verpufft ein wesentlicher Teil der Mittel für den Aufschwung Ost in den alten Bundesländern.

- Selbst die Mittel, die über öffentliche Aufträge vergeben werden und insofern durchaus zu steuern wären, gehen bis heute in erheblichem Umfang an Unternehmen aus dem Westen; eine Quotierung ist bis heute nicht durchgesetzt worden.

- Trotz all dieser Fördermaßnahmen werden die Grenzen der herkömmlichen Wirtschaftsförderung erreicht. Es wird deutlich, daß die Unternehmen auch bei z. T. extremen Investitionszulagen und Zuschüssen nicht investieren (können), wenn die Nachfrage nach den Produkten nicht gegeben ist. Die Folgen und Konsequenzen werden gegenwärtig deutlich, wenn wesentliche Unternehmen ihre Investitionen in den neuen Bundesländern absagen, verschieben oder deutlich kürzen. 
Neue Buchpublikation

\section{Blickwende in der Technologiepolitik}

Die Publikation ,Blickwende in der Technologiepolitik' umfaßt den Endbericht des Forschungsprojektes ,Chancen und Risiken einer auf regionale Bedürfnisse ausgerichteten Tech nologiepolitik", welches im Rahmen des Programms ,Mensch und Technik - Sozialverträgliche Technologiepolitik' des Landes Nordrhein Westfalen gefördert wurde. In dem vom Projektteam - es bestand mehrheitlich aus Mitarbeitern des IÖW Berlin/Wuppertal - in zwei jähriger Tätigkeit erstellten Konzept einer regional- und bedürfnisorientierten Technologiepolitik wird eine Blickwende in der Technologiepolitik vollzogen. Auf der Basis eines kritischen Technikverständnisses werden für einzelne Handlungsfelder integrierte technologie-, struktur- und regionalpolitische Handlungsoptionen erarbeitet und in der Referenzregion, Bergisches Land' iberprït. Der theoretische Ansatz des Projektes läßt sich wie folgt charakterisieren:

- Ausgangspunkt sind nicht Techniken, die es - zumindest nachträglich - sozial, ökologisch und arbeitnehmerfreundlich zu gestalten gilt, sondern manifeste Bedürfnisse, die befriedigt, und Probleme, die gelöst werden sollen. Das bedürfnisorientierte Technikverständnis eröffnet Perspektiven, die über den, instrumentellen Anteil ' des Umgangs mit den Menschen und der Natur hinausgehen und auch die ,Art und Weise dieses Umgangs und dessen stoffliche, soziale und informationelle Grundlagen reflektieren.
- Einbezogen in die perspektivischen Überlegungen zur Regionalentwicklung werden nicht nur die ökonomischen Potentiale der Industrie und des Dienstleistungssektors, sondern auch die Landwirtschaft und die Hauswirtschaft, nicht nur die marktförmig organisierte Erwerbswirtschaft, sondern auch die Gemein- und Eigenwirtschaft.

- Der Blick ist zunächst nicht auf den Weltmarkt gerichtet, sondern in die Region, um deren Eigenheiten, Potentiale und Probleme kennenzulernen, ihre spezifischen Stärken und Schwächen wahrzunehmen.

Die Vorschläge für eine angepaßte und schonende Nutzung von endogenen Potentialen beinhalten sowohl stofflich-technische Innovationen als auch soziale Innovationen hinsichtlich der Produktionsorganisation und der Art der Bedürfnisbefriedigung. Konkret werden folgende projektbezogene Entwicklungsvorschläge unterbreitet: ein regionalwirtschaftliches Konzept zur Stärkung der Landwirtschaft am Beispiel des Flachsanbaus und der Weiterentwicklung der Flachsfasern;

- ein Konzept zur Vermeidung, Verlagerung und Optimierung des Verkehrs, das dem schienengebundenen öffentlichen Personenund Güterverkehr im Form einer, Bergischen Regionalbahn' ein zentrale Rolle zuweist;

ein Konzept zur nachhaltigen Pflege des regionalen Wasserpotentials, mit der Option, dieses ,Gold' des Bergischen Landes durch
Verkauf in die nahen Ballungsgebiete der Rheinschiene zu einem Wirtschaftsfaktor zu machen;

- ein Konzept für eine Gesellschaft für eigenständige Regionalentwicklung (GERBEL), die als Klammer zwischen verschiedenen Akteuren und Handlungsfeldern agieren soll. Vor dem Hintergrund der technologie- und strukturpolitischen Konzeption sowie der Vorschläge für einzelne Handlungsfelder werden in einem dritten Schritt Gestaltungsszenarien für eine stärkere Regionalentwicklung entworfen und diese unterschiedlichen Rahmenbedingungen ausgesetzt. So entsteht ein Bild divergierender Möglichkeiten, verschiedener Pfade und Zukunftsoptionen, zu denen sich die Menschen der Region eine Meinung bilden können. Damit werden die Szenarien selber zu einem wichtigen technologiepolitischen Instrument.

Die Erarbeitung und öfentliche Diskussion konkurrierender Entwicklungspfade - und schließlich auch die Entscheidung unter ihnen ist nach Auffassung der Autoren ein wesentliches Element, um die Wirtschaftsstrukturpolitik und die Forschungs- und Technologiepolitik und damit die Auseinandersetzungen über Kernfragen der zukünftigen Bedingungen in der Region - zu demokratisieren.

Arnim v. Gleich, Rainer Lucas, Ruggero Schleicher, Otto Ulrich: Blickwende in der Technologiepolitik. Naturumgang, Bedürfnisse und räumliche Nähe - Ausgangspunkte für Entwicklungsperspektiven der Region Bergisches Land. Westdeutscher Verlag Opladen 1992, 530 S., DM 78,-. Das Buch kann auch über das IÖW bezogen werden.

\section{Konsequenzen aus den bisherigen Überlegungen}

Zusammengefaßt läßt sich die derzeitige Situation wie folgt charakteriseren:

Es herrscht in den neuen Ländern Ratlosigkeit über die weiter zu unternehmenden Schritte; die vermeintlich klassische Therapie greift nicht und insbesondere werden die Wirkungen auf dem Arbeitsmarkt nicht den Notwendigkeiten entsprechen.

Es sind Konzepte gefragt, die Lösungsansätze für diese Problemlagen aufzeigen können. Damit werden grundsätzlich auch Wege eines ökologischen Umbau- bzw. Aufbauprogramms wieder denkbar, die aufgrund der völligen Fehleinschätzungen in der Realität des wirtschaftlichen Vereinigungsprozesses untergegangen sind.

- Grundsätzlich scheint die Offenheit für , alternative ${ }^{6}$ Entwicklungen zuzunehmen, allerdings sind damit keineswegs unbedingt grüne Vorstellungen verbunden.

Die entscheidende Folgerung daraus ist, daß die gegenwärtige Offenheit genutzt wird, um entsprechende umweltorientierte Überlegungen und möglicherweise auch industriepolitische Vorstellungen weiterzuentwickeln. Es wird deutlich, daß die Vorstellung von blühenden Landschaften nicht ausreicht, damit entsprechende Entwicklungen auch eintreten. Mit dieser Vorstellung war und ist verbunden, daß , business as usual ' betrieben werden könne, die Wirtschaft in den neuen Bundesländern sich allein durch die angewandten Förderinstrumentarien entwickeln könne und der Staat nur die Rahmenbedingungen festlegt, aber kein Leitbild für die wirtschaftliche Entwicklung vorgibt.

Die dauerhafte Betonung der Regierungspolitiker, daß die Vereinigung aus der Portokasse $\mathrm{zu}$ bezahlen sein wird, hat dazu geführt, daß die Bereitschaft zum Teilen, wie sie in den Anfangszeiten bestanden hat, nicht genutzt wurde. Die mittlerweile eingetretenen Verdrossenheit infolge der unklaren Vorstellungen der Politik, die disparaten Maßnahmen und die nicht vorhandene Entwicklungsstrategie machen es nunmehr schwer, die Notwendigkeiten zu vermitteln. Die Flickschusterei, das Springen der Politik von Notmaßnahme zu Notmaßnahme, die dauerhafte Unsicherheit hinsichtlich der weiteren wirtschaftlichen Perspektiven - auch in den Unternehmen - lassen einen großen Wurf unwahr- scheinlich erscheinen. Es erscheint wenig sinnvoll, weiterhin allein auf die finanziellen Anreizinstrumente setzen zu wollen, vielmehr drängt die Entwicklung in den neuen Bundesländern geradezu nach neuen Herangehensweisen.

Es stellt sich die Frage, ob eine ökonomische Entwicklung in Ostdeutschland ohne eine neue Leitidee vorankommen kann. Gegenwärtig stellt sich die Situation so dar, daß auf lange Frist die Chancen einer eigenständigen Entwicklung eher begrenzt sind. Die Wahrscheinlichkeit ist groß, daß die ,Ankoppelung ${ }^{66}$ der neuen Bundesländer auf quasi autonomem Weg kaum zu erwarten ist, gegenwärtig fehlen dazu alle Anzeichen. Die Strategie der Erhaltung der industriellen Kerne dürfte an diesem Dilemma wenig ändern. In dieser Situation scheint es notwendig zu sein, zwei Dinge miteinander zu verknüpfen: die wirtschaftliche Entwicklung in den neuen Bundesländern mit den Herausforderungen der globalen Umweltkrise. Dies hätte zwei Vorteile: auf der einen Seite wird immer deutlicher, daß ohne eine Industriepolitik sich in den neuen Ländern wenig bewegen wird, auf der anderen Seite muß die wirtschaftlichen Entwicklung an den Herausforderungen der Umweltproblematik orientiert sein. Dies sollte dazu führen, daß eine 
ökologisch orientierte Industriepolitik in den neuen Bundesländern Platz greift, die Schritte z. B. in Richtung auf die vielbeschworene Effizienzrevolution einschlagen sollte. Dies wäre zumindest ein Ansatzpunkt für die Wirtschaftsund Technologiepolitik. Es ist zwar bekannt, daß mit einer Effizienzrevolution allein die Umweltprobleme nicht gelöst werden können; viel mehr bedarf es u. a. auch weitgehender kultureller Veränderungen. Aber in einer Situation wie in den neuen Bundesländern muß insbesondere der wirtschaftlichen und sozialen Stabilisierung Vorrang eingeräumt werden. Ein Beispiel für eine solche Orientierung konnte Greenpeace geben, das mit seiner Kampagne für den FCKW-freien Foron-Kühlschrank zum einen ein Unternehmen in den neuen Bundesländern rettete und zum anderen damit auch Anstöße nach Westen geben konnte. Und diese $\mathrm{Zu}$ sammenarbeit wird weiter anhalten - Greenpeace wird die Weiterentwicklung des Unternehmens begleiten. Gerade dieses Beispiel macht eines deutlich: durch die Aktionen von Greenpeace wurde die Nachfrage nach diesem umweltfreundlicheren Produkt deutlich erhöht und die Bereitschaft, dieses Unternehmen zu stützen, war und ist hoch

Ein weiteres Beispiel könnte der Wohnungsbau sein: So stellt sich die Frage, warum z. B. im noch immer darniederliegenden Wohnungsbau nicht sehr viel stärker eine ökologische - sprich vor allem eine energieeffiziente Förderstrategie - eingeschlagen wird, die zwar zunächst teuerer sein kann, perspektivisch aber ein-spezielles Know-How in den neuen Ländern aufbauen würde. Gleichzeitig wäre dies ein wichtiger Baustein für die Reduktion der $\mathrm{CO}_{2}$-Emissionen, denn der Aufbau des Wohnungsbestandes hat eine langfristige Orientierung, da hierdurch die Energieverbrauchstrukturen der nächsten 50 Jahre festgelegt werden. Dabei greift die vielfach geäußerte Forderung nach der Verkürzung der Planungsverfahren und nach Deregulierung zu kurz. In diesen Bereichen, die durchaus auch der sonst vielzitierten staatlichen $\mathrm{Da}$ seinsvorsorge unterworfen werden sollten, ist vielmehr frühzeitig die Internalisierung der externen Kosten zu fordern, was eben auch heißt, in den planerischen Verfahren dies zu erzwingen und es nicht dem Spiel des Marktes zu überlas- sen, welche Wärmestandards erforderlich sind bzw. sich durchsetzen.

Eine solche. Wirtschaftspolitik muß kein utopischer Weg sein: Wie die Analyse des wirtschaftlichen Strukturwandels in ökologischer Hinsicht von Martin Jänicke (siehe hierzu auch Seite 22) gezeigt hat, waren die Entlastungseffekte einer solchen Politik in dem Lande Japan - am größten, in dem eine aktive Wirtschaftsstrukturpolitik betrieben wurde. In Japan wurde auch nicht von einer massiven Beeinflussung der Wirtschaftsstrukturen halt gemacht - es wurden direkt Wirtschaftssektoren gefördert, die weniger umweltbelastend waren. Dies ist ein Hinweis darauf, daß es durchaus Handlungsoptionen gibt, die als Kern eine aktive ökologisch orientierte Strukturpolitik zum Inhalt haben. Eine solche Option insbesondere für die neuen Bundesländer - zu entwickeln, ist die Herausforderung für die Gesellschaft der Bundesrepublik, zu deren Bewältigung auch ein aktives Eingreifen der Staates erforderlich sein wird.

Ulrich Petschow, Berlin

\section{Industrielle Beziehungen und Ökologie unter veränderten Rahmenbedingungen}

Alle Überlegungen darüber, ob und in welchem Maße das bestehende System der industriellen Beziehungen in der Bundesrepublik Deutschland eine zunehmende Bedeutung im Umweltschutz haben könnte, müssen drei Ausgangsbedingungen berücksichtigen:

Erstens, daß weder die Gesetze der Betriebsverfassung und der Mitbestimmung ökologische Sachverhalte explizit berücksichtigen, noch die Umweltschutzgesetze die Institutionen der industriellen Beziehungen als Umsetzer und Kontrolleur von Regelungen und Normen einbeziehen. Zweitens ist die Verursachungskette von unternehmensbedingten Umweltproblemen so ausgebildet, daß entsprechende Gegenmaßnahmen in die Kompetenz des Managements und der entsprechenden Funktionsbereiche (Einkauf, Konstruktion, Materialwirtschaft, Sicherheit) fallen. Eine Zuständigkeit der betrieblichen Interessenvertretungen und der Gewerkschaften wird grundsätzlich von den Unternehmensleitungen nicht gesehen.

Drittens tun sich auch die Beschäftigten, die betrieblichen Interessenvertretungen und die $\mathrm{Ge}$ werkschaften schwer, aus eigener Initiative im Umweltschutz aktiv zu werden. Aufgrund der Konzentration auf Betriebspolitik - und d.h. gleichzeitig die Überlassung von Themenfeldern wie lokale Lebensqualität und Verbraucherpolitik an andere soziale Bewegungen -, aufgrund der Focussierung der Interessenpolitik auf Arbeitsplatz- und Einkommenssicherung und de- ren Einbindung in den gemeinsamen Produktivitäts- und Sozialpakt mit dem Unternehmen gibt es systematische Hemmschwellen für ein offensives gewerkschaftliches Verhalten im Umweltschutz. Die Zuständigkeit wird eher beim Staat und die Kompetenz in diesem Feld eher bei der Ökologiebewegung gesehen, zu der die Gewerkschaften notwendigerweise ein ambivalentes Verhältnis haben.

Die Beobachtung der Regulierung unternehmensverursachter Umweltschäden und des Verhaltens der Unternehmensverbände und der Gewerkschaften hat deutlich gemacht, daß bei diesen Wirtschaftsakteuren ganz unterschiedliche und widersprüchliche Anknüpfungspunkte eine Rolle spielen und daß das traditionelle Regelungssystem nicht in der Lage ist, die neuartigen Umweltprobleme angemessen zu bearbeiten. Im Unternehmerlager haben sich drei Gruppen von Unternehmen herausgebildet: die größer werdende Gruppe der Vorreiter, die sich durch ein ökologisches Management eine höhere Akzeptanz des Unternehmens und der Produkte erhoffen; die größere Gruppe der stillen Anpasser an die gesetzlich vorgeschriebenen Standards; und die schwer zu quantifizierende Gruppe der Unternehmen, die solche Anpassungsmaßnahmen auf verschiedenste Weise hinauszuzögern oder zu vermeiden versuchen. Auch nach der neuesten Unternehmensbefragung (FUFF 1991) sind die ökonomischen Anreize des Umweltschutzes (Kostensenkung, Ertragssteigerung) bis- her nur schwach wirksam. Wichtiger ist die langfristige Risikoperspektive, die sich im Bedeutungszuwachs sog. weicher Faktoren ausdrückt (Ansehen in der Öffentlichkeit, Wahrnehmung sozialer Verantwortung, Mitarbeitergewinnung und -motivation). Im Gegenteil, als Unternehmensziele, die am stärksten durch steigende Umweltschutz-Anforderungen gefährdet sind, wurden Gewinnerzielung und Liquidität genannt. Die Betriebsräte sind durch die steigenden Umweltschutz-Anforderungen direkt wenig betroffen; sie reagieren i.d.R. auf die Unternehmenspolitik in diesem Feld. Wird das Unternehmen durch die Anforderungen hoch belastet und reagiert mit Opposition, dann sieht der Betriebsrat die Beschäftigung gefährdet. Reagiert das Unternehmen dagegen offensiv und präventiv auf die Anforderungen, können die Betriebsräte dieser Strategie folgen, und auf Arbeitsplatzsicherung und Verbesserungen im Arbeits- und Gesundheitsschutz hoffen. Die Gewerkschaften stehen stärker in einer gesellschaftlichen Verantwortung, müssen die globalen Umweltrisiken gegenüber den einzelbetrieblichen Sichtweisen in den Vordergrund rücken und auf die veränderten lebensweltlichen Erfahrungen, Orientierungen und Verhaltensweisen zumindest eines Teils der Mitglieder reagieren. Damit ergeben sich unterschiedliche Bezüge zum Umweltthema, die durchaus zu Spannungen und Glaubwürdigkeitsverlusten zwischen Mitgliedern, betrieblichen Interessenvertretungen und Gewerkschaften führen können. Verschärfend kommt hinzu, daß im Umweltschutz die Betriebsgrenze eine entscheidende Rolle spielt: die öffentliche Kritik am Unternehmen soll geschlossen abgewehrt werden, das Publikmachen 
(c) 20I0 Authors; licensee IÖW and oekom verlag. This is an article distributed under the terms of the Creative Commons Attribution Non-Commercial No Derivates License (http://creativecommons.org/licenses/by-nc-nd/3.o/), which permits unrestricted use, distribution, and reproduction in any medium, provided the original work is properly cited. 